\title{
Predictors for voiding trial failure after minimally invasive sacrocolpopexy
}

\begin{abstract}
Introduction: Catheter associated urinary tract infections (CAUTIs) due to indwelling catheters pose a serious health and financial burden in the US. Patients who undergo reconstructive pelvic surgery experience postoperative voiding dysfunction at a significant rate, requiring prolonged catheterization. The preoperative identification of patients at highest risk of failing a backfill trial of void (TOV) would allow for selected instruction in self-catheterization techniques and minimize the prevalence in dwelling catheters.
\end{abstract}

Objective: To identify predictors for voiding trial failure after minimally-invasive (laparoscopic or robot-assisted) sacrocolpopexy for surgical correction of prolapse in patients who did not undergo a concomitant urinary anti-incontinence procedure.

Methods: All subjects undergoing minimally-invasive sacrocolpopexy without concomitant anti-incontinence surgery from October 2006 to July 2010 at our institution were included. Patient characteristics including basic demographics, medical and surgical history, operative characteristics, and baseline preoperative urodynamic testing were included. The data was analyzed with logistic regression for continuous variables and Pearson chi-squared test for dichotomous variables (Stata IC-13).

Results: 56 subjects were identified. Of those, 5 subjects $(8.9 \%)$ failed a postoperative voiding trial. No significant association between examined variables and failure of trial of void were observed. Concomitant vaginal surgery approached significance with a p-value of 0.058 .

Conclusion: The percentage of subjects that failed a voiding trail was comparable to that described in the minimally invasive literature. Providers should educate all patients undergoing minimally invasive sacrocolpopexy as to self-catheterization techniques given the inability to identify preoperative risk factors.

Keywords: voiding trial, failure, sacrocolpopexy, minimally invasive, predictors
Volume 3 Issue 2 - 2015

\author{
Alexcis PThomson,' Caroline E Foust- \\ Wright, ${ }^{2}$ Rebecca Posthuma Batalden, ${ }^{2}$ May \\ MWakamatsu, ${ }^{2}$ Samantha J Pulliam, ${ }^{2}$ Milena \\ MWeinstein ${ }^{2}$ \\ 'Brigham and Womens Hospital/Massachusetts General \\ Hospital, USA \\ 2Department of Obstetrics, Gynecology, and Reproductive \\ Biology, Massachusetts General Hospital, USA
}

\begin{abstract}
Correspondence: Alexcis PThomson,Vincent Obstetrics and Gynecology, Massachusetts General Hospital and Harvard Medical School, 55 Fruit Street Boston, MA, United States, 02II4, Tel 404-909-4442, Fax 617-724-3498, Email athomson2@partners.org
\end{abstract}

Received: October 29, 2015 | Published: November 6, 2015

\section{Introduction}

The healthcare industry is increasingly focusing on reducing preventable complications. Iatrogenic complications such as pressure wounds, central line infections and catheter-associated urinary tract infections (CAUTIs) are not only costly, but can lead to potentially life threatening sequelae. It is estimated that each CAUTI costs anywhere from \$749-1007, and CAUTIs were among the first selected to the non-payment list by Medicare as of October 2008. ${ }^{1}$ In the field of urogynecology, prolonged catheterization is a reality for many patients, especially in the postoperative setting. Postoperative voiding dysfunction after prolapse surgeries with or without incontinence surgery have been estimated between $22-62 \%{ }^{2-5}$ Pelvic organ prolapse affects one in 10 women $^{6}$ and approximately 200,000 patients per year undergo a prolapse surgery in the United States. ${ }^{7}$ Nearly all patients are evaluated for postoperative voiding dysfunction via a backfill trial of void (TOV). In the era of minimally invasive surgery, patients are leaving the hospital earlier, and are often left to deal with an uncomfortable and cumbersome indwelling catheter a potential nidus for infection, especially when being cared for by a non-health professional. Intermittent self-catheterization has been shown to be a superior alternative to indwelling urinary catheters, leading to reduced CAUTIs and increased patient satisfaction. ${ }^{8,9}$ Instituting this policy universally does require a significant investment in patient education on behalf of the healthcare provider, as a lack of understanding or compliance with self-catheterization can lead to disastrous consequences, including permanent bladder injury secondary to irreversible nerve damage. ${ }^{10}$ The ability to risk-stratify patients preoperatively, in terms of individual risk for voiding trial failure, could focus patient education regarding self-catheterization techniques to the highest risk population, more efficiently utilizing healthcare industry resources. There is a paucity of data regarding risk factors for postoperative TOV failure after minimally invasive sacrocolpopexy and, to our knowledge, no studies have focused specifically on preoperative risk factors, available to the healthcare provider at the time of preoperative evaluation, at which time patient education regarding self-catheterization techniques could be implemented.

\section{Materials and methods}

All subjects who underwent a minimally-invasive sacrocolpopexy without concomitant anti-incontinence surgery at the Massachusetts General Hospital from October 2006 to July 2010 were reviewed. This time period was chosen as it included a sample of both robotic and laparoscopic procedures. Patients who underwent a concomitant anti-incontinence procedure, such as placement of a suburethral sling, were excluded, given the propensity for this procedure to affect postoperative voiding.

Electronic medical records were reviewed retrospectively and demographic information was collected in an attempt to identify preoperative factors that may affect postoperative voiding. Variables 
collected included age, body mass index (BMI), length of surgery, history of prior surgery, type of surgery being performed, parity, menopausal status, use of hormonal therapy preoperatively, prolapse stage, history of diabetes, incontinence, constipation, urinary retention, recurrent urinary tract infections, urinary tract infection within one month, history of depression and any use of antidepressants, as well as detailed urodynamic testing results including uroflow, cystometry, abdominal leak point pressure and pressure voiding studies.

Extensive peri- and postoperative details were not collected, as this study was designed specifically to identify preoperative risk factors for failing a trial of void. Length of surgery and intraoperative complication information was collected, however, as these variables often have a large impact on postoperative voiding, although not able to be predicted preoperatively.

All procedures were performed by 1 of 3 urogynecologists at the Massachusetts General Hospital, with each surgeon utilizing both robotic and laparoscopic routes. In all cases, the bladder was backfilled with indigo carmine intraoperatively and dissected off of the anterior vaginal wall. The Y arms of a Y-shaped portion of type 1 macro porous polypropylene mesh were attached to the anterior and posterior vaginal walls using 4 to 6 CVO Gor-tex interrupted sutures, respectively. The peritoneum of the bladder flap was then used to retroperitonealize the mesh.

Postoperatively, the foley catheter was left in situ and all patients underwent a backfill TOV on postoperative day 1 . The bladder was backfilled with $300 \mathrm{~mL}$ of normal saline and the catheter was removed. The patient was allotted 30 minutes to void $200 \mathrm{~mL}$. If the patient was unable to do so, the trial of void was considered failed and the patient had the catheter replaced for a repeat trial of void the next available clinic day, usually within 3 days of discharge from the hospital.

Study data were collected and managed using REDCap electronic data capture tools at the Massachusetts General Hospital. The characteristics of the subjects who failed their trial of void were compared to those of the subjects who passed using multiple logistic regressions for continuous variables and Pearson chi-squared test for dichotomous variables (Stata IC-13). This study was approved by the Partners Institutional Review Board.

\section{Results}

A total of 60 subjects were identified during the study period. After excluding 4 patients who experienced intraoperative complications necessitating prolonged catheterization ( 2 events of sutures in the bladder, a cystotomy and a ureteral kink requiring release), 56 subjects remained; 5 of whom failed a postoperative voiding trial (8.9\%). When examining patient characteristics, such as age, BMI, parity, stage of prolapse, presence of diabetes, depression, chronic constipation or retention, menopause and previous surgeries, no statistical difference was seen between those who failed compared with those who passed the TOV. When examining operative characteristics, including use of a scopolamine patch, route of procedure (robotic vs. laparoscopic), concomitant hysterectomy and vaginal surgery, no statistically significant association was seen. Concomitant vaginal surgery was the only variable that approached significance, with a p-value of 0.058. Preoperative urodynamic information, including cystometric maximum capacity, post-void residual, maximum urethral closure pressure, normal desire to void, peak flow rate, and detrusor voiding mechanism was also collected. Unfortunately, 48 of the 56 patients had incomplete or missing data, and therefore, this information was not included in the analysis.

\section{Discussion}

There are a paucity of data describing voiding trial failure rates among patients undergoing minimally invasive sacrocolpopexy, and even fewer studies examining preoperative risk factors in this specific patient population. In our study of 56 patients, $8.9 \%$ of patients undergoing a minimally invasive sacrocolpopexy failed a backfill TOV on postoperative day 1 . This is consistent with observed voiding trial failure rates following minimally invasive procedures in gynecologic literature $(7.1 \%$ to $18.7 \%) .{ }^{11,12}$ To our knowledge, there exists only one other study specifically describing voiding trial failure rates after minimally invasive sacrocolpopexy. ${ }^{13}$ This retrospective cohort study of 290 patients at a single institution over a 3 year period reported a $27.2 \%$ failure rate, however, this cohort included patients who underwent concomitant mid-urethral slings, which are known risk factors for postoperative urinary retention (and were excluded from our study for this reason specifically). In terms of voiding trial failure rates after vaginal prolapse surgery, rates ranging from $29 \%$ to $32 \%$ have been observed; much higher than those seen in our study. ${ }^{4}$ This is potentially explained by increased edema and tissue destruction surrounding the urethra caused by aggressive dissection and manipulation required by extensive vaginal surgery. These studies also include patients with concomitant anti-incontinence procedures.

The main strength of this study was the focus on risk factors that could be identified at a preoperative visit, such that the patient provider could have the opportunity to teach self-catheterization techniques in order to mitigate the risks associated with an indwelling catheter. Risk factors for voiding trial failure, in general, are multifactorial, including those occurring preoperatively, perioperatively, and postoperatively. Perioperative and postoperative risk factors, such as estimated blood loss, intravenous fluid administered, perioperative and postoperative analgesic and sedative medication administered, extensive surgical dissection resulting in disruption of nerve plexuses, and tissue edema causing obstruction of voiding, have all been described in the literature. ${ }^{2,5,14}$ As these factors are not readily available preoperatively, these were not examined in depth in this study. An attempt was made, however, to look at surrogate markers that may predict some of these risk factors. These include chronic pain syndromes (leading to need for higher doses of analgesic and sedative medication), a history of pelvic surgery and concomitant vaginal surgery, including anterior and posterior repair (contributing to tissue edema and nerve disruption from more extensive dissections or distorted anatomy secondary to scarring), planned concomitant hysterectomy (more surgical steps leading to higher blood loss and intraoperative complications), etc. Other preoperative risk factors examined included age, BMI, menopausal status, parity and prolapse stage, as these can reflect anticipated surgical difficulty.

Our study was unable to find any linkage between the patient variables analyzed and failure of a postoperative voiding trial. Concomitant vaginal surgery did closely approach significance, however, with a p-value of 0.058 . Data are mixed regarding the effect of an anterior repair on voiding trial failure, with some studies noting an association3, while other have been unable to do so4. In the latter study, a higher prevalence of voiding dysfunction in patients with a high grade cystocele and the application of a posterior repair was also been observed. ${ }^{4}$ Geller et al. ${ }^{13}$ examined a wide variety of procedures including vaginal, abdominal, laparoscopic and robotic, while Hakvoort et al. ${ }^{4}$ examined exclusively vaginal procedures, thus, results may not be entirely generalizable to our patient population. In the aforementioned study by Turner et al. ${ }^{13}$ examining preoperative 
risk factors for voiding dysfunction in patients undergoing a minimally invasive sacrocolpopexy, none of the 290 patients who underwent a minimally invasive sacrocolpopexy had a concomitant vaginal surgery, thus the potential for this variable to cause postoperative voiding dysfunction was unable to be analyzed.

A significant proportion of our patients underwent preoperative urodynamic testing, and each was determined not to need a concomitant anti-incontinence procedure on this basis, as well as by history and physical exam. Unfortunately, this information was missing in enough patients to be deemed unsuitable to include in the analysis. While it is well documented that preoperative urodynamic abnormalities do not predict postoperative voiding dysfunction, preoperative voiding dysfunction is a known predictor, and by eliminating the subset of these patients with this a priori confounder, our results are strengthened. . $^{10,15,16}$

The major limitation of this study is small sample size. Any statistical significance is difficult to achieve with a small patient population, thus, repeating this pilot study with a larger cohort would be beneficial. In addition, as subjects undergoing concomitant antiincontinence procedures were excluded, our results may not be generalizable to that specific patient population. Another inherent limitation of this study is the multi-factorial nature of postoperative voiding trial failure, as previously mentioned. Without the ability to accurately predict and quantify many perioperative and postoperative variables, it is very difficult to assess the impact of these variables on the results of our study.

\section{Conclusion}

The percentage of patients who failed a postoperative backfill TOV in our study is similar to that described in minimally invasive literature. No preoperative variables demonstrated a statistically significant association with voiding trial failure; however concomitant vaginal surgery did closely approach significance. Given the lack of ability to accurately predict who will fail a postoperative trial of void at this time, all patients should be counseled regarding selfcatheterization techniques preoperatively. Further studies should be pursued including larger sample sizes in order to better identify statistically significant associations between preoperative variables and voiding trial failure.

\section{Acknowledgments}

None.

\section{Conflicts of interest}

The authors declare there is no conflict of interests.

\section{References}

1. Meddings J, Rogers MA, Krein SL, et al. Reducing unnecessary urinary catheter use and other strategies to prevent catheter-associated urinary tract infection: an integrative review. BMJ Qual Saf. 2014;23(4):277-289.
2. Book NM, Novi B, Novi JM, et al. Postoperative voiding dysfunction following posterior colporrhaphy. Female Pelvic Med Reconstr Surg. 2012;18(1):32-34.

3. Geller EJ, Hankins KJ, Parnell BA, et al. Diagnostic accuracy of retrograde and spontaneous voiding trials for postoperative voiding dysfunction: a randomized controlled trial. Obstet Gynecol. 2011;118(3):637-642.

4. Hakvoort RA, Dijkgraaf MG, Burger MP, et al. Predicting short-term urinary retention after vaginal prolapse surgery. Neurourol Urodyn. 2009;28(3):225-228.

5. Walter PJ, Dieter AA, Siddiqui NY, et al. Perioperative anticholinergic medications and risk of catheterization after urogynecologic surgery. Female Pelvic Med Reconstr Surg. 2014;20(3):163-167.

6. DeLancey JO. The hidden epidemic of pelvic floor dysfunction: achievable goals for improved prevention and treatment. Am J Obstet Gynecol. 2005;192(5):1488-1495.

7. Boyles SH, Weber AM, Meyn L. Procedures for pelvic organ prolapse in the United States, 1979-1997. Am J Obstet Gynecol. 2003;188(1):108-115.

8. Turi MH, Hanif S, Fasih Q, et al. Proportion of complications in patients practicing clean intermittent self-catheterization (CISC) vs indwelling catheter. JPMA J Pak Med Assoc. 2006;56(9):401-404.

9. Woodward S, Steggal M, Tinhunu J. Clean intermittent self-catheterisation: improving quality of life. Br J Nurs Mark Allen Publ. 2013;22(9):S22-S25.

10. Tammela T, Kontturi M, Lukkarinen O. Postoperative urinary retention. I. Incidence and predisposing factors. Scand J Urol Nephrol. 1986;20(3):197201.

11. Liang CC, Lee CL, Chang TC, et al. Postoperative urinary outcomes in catheterized and non-catheterized patients undergoing laparoscopic-assisted vaginal hysterectomy--a randomized controlled trial. Int Urogynecol J Pelvic Floor Dysfunct. 2009;20(3):295-300.

12. Smorgick N, DeLancey J, Patzkowsky K, et al. Risk factors for postoperative urinary retention after laparoscopic and robotic hysterectomy for benign indications. Obstet Gynecol. 2012;120(3):581-586.

13. Turner LC, Kantartzis K, Shepherd JP. Predictors of postoperative acute urinary retention in women undergoing minimally invasive sacral colpopexy. Female Pelvic Med Reconstr Surg. 2015;21(1):39-42.

14. Keita H, Diouf E, Tubach F, et al. Predictive factors of early postoperative urinary retention in the postanesthesia care unit. Anesth Analg. 2005;101(2):592-596.

15. Fitzgerald MP, Kulkarni N, Fenner D. Postoperative resolution of urinary retention in patients with advanced pelvic organ prolapse. Am J Obstet Gynecol. 2000;183(6):1361-1363.

16. Sanses TV, Brubaker L, Xu Y, et al. Preoperative hesitating urinary stream is associated with postoperative voiding dysfunction and surgical failure following Burch colposuspension or pubovaginal rectus fascial sling surgery. Int Urogynecology J. 2011;22(6):713-719. 